\title{
Preface of the special issue on "Percutaneous endoscopic system for spinal diseases"
}

\author{
Hisashi Koga ${ }^{1,2}$ \\ ${ }^{1}$ Department of Neurosurgery and Orthopaedics, Iwai Orthopaedic Medical Hospital, Edogawa-ku, Tokyo 133-0056, Japan. \\ ${ }^{2}$ Department of Orthopaedic Surgery, Inanami Spine and Joint Hospital, Shinagawa-ku, Tokyo 140-0002, Japan.
}

Correspondence to: Dr. Hisashi Koga, Department of Orthopaedics, Iwai Orthopaedic Medical Hospital, 8-17-2 Minamikoiwa, Edogawa-ku, Tokyo 1330056, Japan. E-mail: hkoga0808@gmail.com

How to cite this article: Koga H. Preface of the special issue on "Percutaneous endoscopic system for spinal diseases". Mini-invasive Surg 2017;1:41-2.

Article history: Received: 13-04-2017 Accepted: 14-04-2017 Published: 30-06-2017

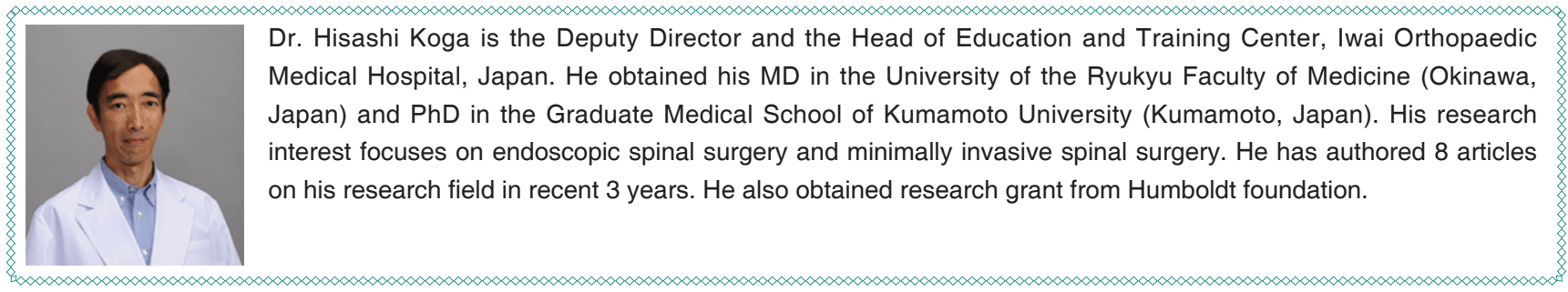

It is our great privilege to present the special issue of Mini-invasive Surgery, an open-access journal devoted to exploring many contemporary issues affecting minimally invasive surgery. The first special issue of Mini-invasive Surgery is intended to introduce the latest advancement of percutaneous endoscopic lumbar discectomy (PELD), which is a single portal full-endoscopic system originally developed for the treatment of lumbar disc herniation (LDH). As PELD has been rapidly growing in East Asia (China, Korea, and Japan), we invited submissions from these countries. The majority of the articles are from Japan. However, articles presented in this issue cover all recent advancements in the field of PELD.

PELD technique has been developed along with the advancement of operative instruments. In particular, the development of a high-speed drill used in narrow and long endoscopic lumen has expanded not only target spinal diseases, but also the operative spinal area. Currently, we are already able to successfully treat cervical radiculopathy (Ohmori et al.), lumbar spinal canal stenosis (Ohara et al.), LDH with narrow interlaminar space (Koga et al.), and cervical spinal canal stenosis (Nishimura) with PELD. However, this technique does have some limitations, which must be recognized and we must utilize the supportive materials such as free-running electromyogram monitoring (Kitahama et al.) to secure safety of the technique.

The technique of PELD requires some specific technical and anatomical considerations that will be

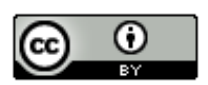
This is an open access article licensed under the terms of Creative Commons Attribution 4.0 International License (https://creativecommons.org/licenses/by/4.0/), which permits unrestricted use, distribution, and reproduction in any medium, as long as the original author is credited and the new creations are licensed under the identical terms.

For reprints contact: service@oaepublish.com

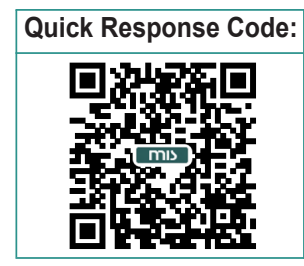


addressed in this special issue of Mini-invasive Surgery. For example, the initial stage of PELD is a completely blind maneuver. Therefore, anatomical knowledge is imperative (Sakane) along with accurate preoperative measurement using radiological data (Kitahama et al.). In addition, the appropriate preoperative diagnosis remains extremely important. Accordingly, we asked Dr. Kim to submit review article regarding differential diagnosis of LDH. From each of these articles, we hope that readers with varying levels of experience with PELD can utilize this issue, from the beginner to the expert who can imagine new operative approaches.

We also have emphasized that the technical difficulties associated with this procedure and a lack of a steep learning curve. Therefore, strict training programs under the guidance of expert surgeons is essential for the expansion of PELD. We propose that the establishment of a training system is the most important factor for safely disseminating PELD. From this viewpoint, we would like to introduce the wellestablished training system from Japan for orthopedic surgeons (Dezawa), for neurosurgeons (Mizuno) and from China (Yang). These systems are anticipated to be disseminated to other countries to assist spinal surgeons with mastering PELD.

We would like to acknowledge various people who have contributed to the completion of this issue, with special mention to our co-Guest Editors: Dr. Kyongsong Kim
(Department of Neurosurgery, Chiba Hokuso Hospital, Nippon Medical School) and Dr. Manabu Sasaki (Department of Neurosurgery and Spine Surgery, Iseikai Hospital, Osaka, Japan) and Assistant Editor: Ms. Anne Niu. Without their tireless efforts in editing and personal sacrifice, this special issue would not have been published. We would also like to acknowledge our publisher, OAE publishing Inc., for their support of this international special issue. Finally we also express our appreciation and thanks to the Japanese Spine Surgical Societies (Orthopaedic and Neurosurgical) and the special communities of these societies (Japan PED study group, Less-Invasive and Endoscope Spinal Neurosurgery) who deeply recognize the importance of minimally invasive spinal surgery.

\section{Authors' contributions}

H. Koga contributed solely to this preface.

Financial support and sponsorship

None.

\section{Conflicts of interest}

There are no conflicts of interest.

\section{Patient consent \\ Not applicable.}

Ethics approval

Not applicable. 